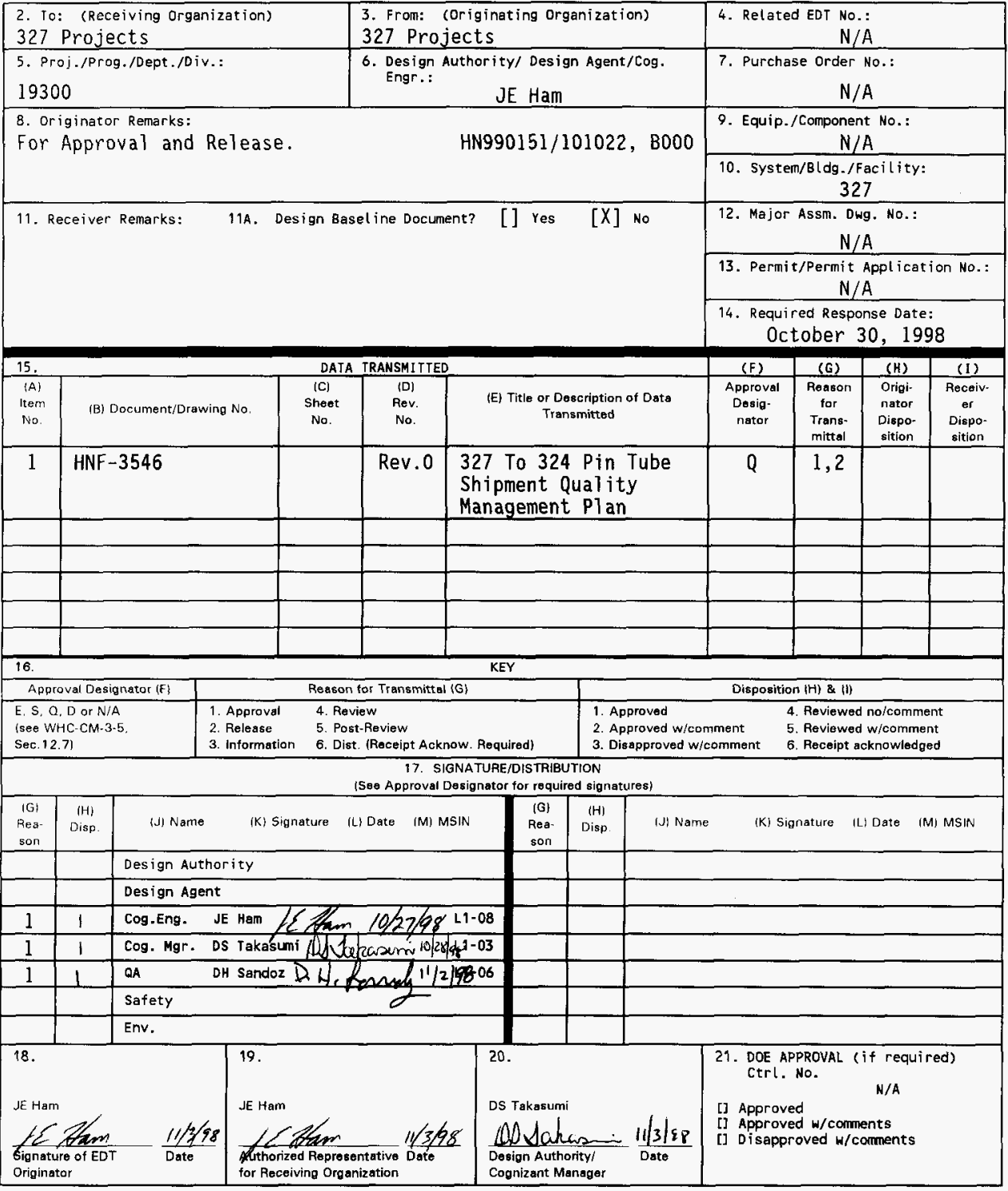

BD-7400-172-2(05/96) GEF09? 


\title{
327 TO 324 PIN TUBE SHIPMENT QUALITY MANAGEMENT PROCESS PLAN
}

\section{J.E. Ham}

Babcock and Wilcox Hanford Co., Richland, WA 99352

U.S. Department of Energy Contract DE-ACO6-96RL13200

\author{
EDT/ECN: 616491 \\ Org Code: 19300 \\ UC: 513 \\ B\&R Code: EW7002010 \\ Charge Code: HN990151 \\ Total Pages: 5
}

Key Words: 327 Facility, 324 Facility, Pin Tube, Shipment, Serf Cask, Legacy, Pins, Drillings

Abstract: The B\&W Hanford Company's (BWHC) 327 Facility, in the 300 Area of the Hanford Site, is preparing to ship five Pin Tubes to the 324 Facility for storage and eventual dispostion. The Pin Tubes consist of legacy fuel pin pieces and drillings. They will be over-packed in new Pin Tubes and transported to 324 in three shipments. Once received at 324 , two of the shipments will be combined for storage as a fissionable material batch, and the other shipment will be added to an existing batch.

\footnotetext{
TRADEMARK DISCLAIMER. Reference herein to any specific comercial product, process, or service by trade name, trademark, manufacturer, or otherwise, does not necessarily constitute or imply its endorsement, recommendation, or favoring by the united States Government or any agency thereof or $i$ ts contractors or subcontractors.
}

Printed in the United States of America. To obtain copies of this document, contact: Document Control Services, P.O. Box 950, Mailstop H6-08, Richland WA 99352, Phone (509) 372-2420; Fax (509) 376-4989.
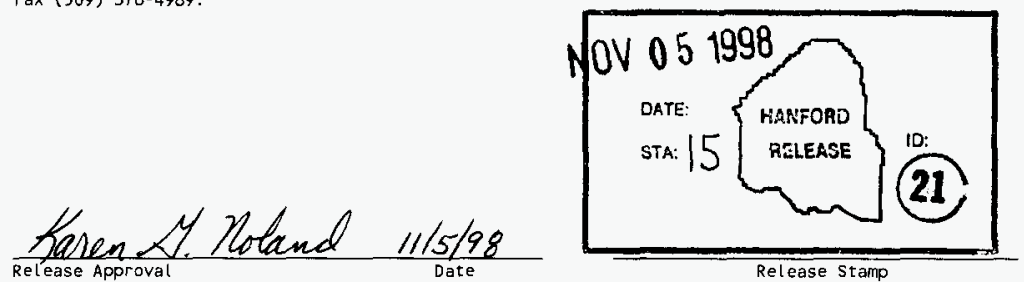

\section{Approved for Public Release}




\subsection{PURPOSE}

The purpose of this Quality Management Plan is to ensure appropriate and effective quality assuring activities have been built into the work controlling documents and procedures.

\section{$2.0 \mathrm{SCOPE}$}

The scope of this project involves the movement, over-packing, and shipment of five Pin Tubes from the 327 Building to the 324 Building. The Pin Tubes consist of legacy fuel sections or scrap including drillings, and will be transported using the SERF Cask in three shipments. The material will be received at the 324 Facility, where it will be transferred to D-Cell and stored in two fissionable material batches (one existing and one new). Babcock \& Wilcox Hanford Company (BWHC) is responsible for both the 324 and 327 Facilities, and therefore, all activities under this project. DynCorp Tri-Cities will provide transportation of the casks between the facilities.

\subsection{OVERVIEW}

Five Pin Tubes are located throughout 327 in two canyon cells and the storage basin. Three of the Pin Tubes are currently stored underwater in the 327 Storage Basin. One Pin Tube is located in A-Cell and another is in SERF Cell. The three Pin Tubes stored in overpack tubes in the basin will be moved to A-Cell one at a time for identification and verification to the set criteria ( $<50 \mathrm{ml}$ liquid in overpack tube and Pin Tube damage free). The overpack tubes will be removed and discarded. A pre-marked Pin Tube Overpack will be matched with each Pin Tube, and then the Pin Tube placed into the Overpack for shipment. The shipments will be designated as Shipments A, B and C. The three basin Pin Tubes are to be loaded and shipped separately from each other due to the fissionable material limits in the SERF Cask.

The Pin Tube currently located in A-Cell, and the Pin Tube located in SERF Cell will be loaded into the SERF Cask as a part of Shipment $\mathbf{C}$. These two Pin Tubes will not be checked for liquid because they have not been stored underwater. However, each Pin Tube will undergo identification verification, and will be over-packed in new Pin Tube Overpacks.

Once transported to the 324 Facility, the Pin Tube Overpack IDs will be verified in the Radiochemical Engineering Complex (REC) Airlock, and will be transferred to one of two locations (batches) in D-Cell. Shipment A will be added to an existing fissionable material batch located in the Southwest corner of D-Cell, and Shipments B and C will be combined to form a new fissionable material batch in D-Cell 


\subsection{PROCESS PLAN}

\section{Basin Pin Tube To SERF Cask (Shipment A, B, and C)}

The three Pin Tubes stored in the basin will be removed from the basin one at a time, and shipped separately as Shipments A, B, and C. Shipments A and B will each contain only one over-packed Pin Tube for transport. Shipment $C$ will be joined by two other over-packed Pin Tubes, one from A-Cell and one from SERF Cell. Each Pin Tube will be transferred, and prepared for transport in the following manner.

The over-packed Pin Tube is transferred to A-Cell. The Pin Tube overpack identification is reviewed and recorded. The swagelok'cap will be removed from the overpack, and any liquid will be drained into an empty container and transferred into a graduated cylinder for measurement. The amount of liquid and the Pin Tube will be examined against set criteria $(<50-\mathrm{ml}$ liquid and damage free). A verification will be made that the criteria have been met. A new Pin Tube Overpack, which has been premarked with an identification number will be matched with the Pin Tube and verified. Quality Control will verify the Pin Tube and Overpack IDs. Then, the Pin Tube will be placed into the Overpack. A Swagelok fitting will be installed and the Overpack loaded into the SERF Cask.

\section{A-Cell \& SERF Cell Pin Tubes To SERF Cask (Shipment C)}

Once the basin Pin Tube for Shipment $C$ has been loaded into the SERF Cask, the ACell Pin Tube will be prepared and loaded. The SERF Cask will then be transferred to SERF Cell where another Pin Tube will be prepared and loaded. Shipment $C$ will then be ready for transport to the 324 Facility. Both Pin Tubes will be prepared and transferred to the SERF Cask in the following manner.

The Pin Tube identification will be verified. A new Pin Tube Overpack, which has been pre-marked with an identification number will be matched with the Pin Tube and verified. Quality Control will verify the Pin Tube and Overpack IDs. Then, the Pin Tube will be placed into the Overpack. A Swagelok fitting will be installed, and the Overpack loaded into the SERF Cask

\section{Ship Pin Tubes From 327 To 324 D-Cell}

Documentation and shipping papers will be reviewed by the 327 Facility Material Balance Area (MBA) Custodian, and then verified by Quality Control prior to transport from 327 to 324 . Once received, the 324 Facility MBA Custodian will review the shipping papers and approve the unloading of the shipment in the Radiochemical Engineering Complex (REC) Airlock.

\footnotetext{
* Swagelok is a trademark of Crawford Fitting Company, Solon, OH.
} 
The Pin Tube Overpack identifications will be verified in the REC Airlock. Shipment A will have a lifting cradle (collar) pre-installed at 327, and will be added to an existing fissionable material batch in D-Cell. Shipments $B$ and $C$ will be placed in another lifting cradle (metal box) and will become a new fissionable material batch in DCell. Once the storage location is reviewed and verified, the cradle will be transported to its designated location in D-Cell via an overhead crane. The criticality postings will be updated and verified

Table 1.

\begin{tabular}{|c|c|c|c|c|c|}
\hline Activity & Product & $\begin{array}{c}\text { Work Control } \\
\text { Method } \\
\text { JCS No. }\end{array}$ & $\begin{array}{c}\text { Procedure } \\
\text { and } \\
\text { Instruction }\end{array}$ & $\begin{array}{c}\text { Lead } \\
\text { Individual } \\
\text { (Title \& Co.) } \\
\end{array}$ & $\begin{array}{l}\text { Quality } \\
\text { Assuring } \\
\text { Actions }\end{array}$ \\
\hline \multicolumn{6}{|c|}{327 Basin Pin Tube To SERF Cask (Shipment $A, B$, and $C$ ) } \\
\hline $\begin{array}{l}\text { Transfer Pin } \\
\text { lube from Basin } \\
\text { to A-Cell }\end{array}$ & $\begin{array}{l}\text { Material } \\
\text { movement }\end{array}$ & N/A & $\begin{array}{l}\text { 3M-SOP-PTL- } \\
084,-118\end{array}$ & $\begin{array}{l}\text { HCT Supervisor, } \\
\text { BWHC }\end{array}$ & $\begin{array}{l}\text { HCT signs } \\
\text { CFMl }\end{array}$ \\
\hline $\begin{array}{l}\text { Review Pin Tube } \\
\text { overpack ID and } \\
\text { record }\end{array}$ & Proper selection & N/A & 3M-TWP-98-017 & $\begin{array}{l}\text { HCT Supervisor, } \\
\text { BWHC }\end{array}$ & $\begin{array}{l}\text { Work plan step } \\
\text { PIC Initial/date }\end{array}$ \\
\hline $\begin{array}{l}\text { Open Pin Tube } \\
\text { overpack and } \\
\text { drain liquid }\end{array}$ & $\begin{array}{l}\text { Ready for } \\
\text { examination }\end{array}$ & $\mathrm{N} / \mathrm{A}$ & 3M-TWP-98-017 & $\begin{array}{l}\text { HCT Supervisor, } \\
\text { BWHC }\end{array}$ & Work plan steps \\
\hline $\begin{array}{l}\text { Examine Pin } \\
\text { Tube \& Verify } \\
\text { criteria met }\end{array}$ & $\begin{array}{l}\text { Pin Tube dry and } \\
\text { undamaged }\end{array}$ & $\mathrm{N} / \mathrm{A}$ & $3 M-T W P-98-017$ & $\begin{array}{l}\text { HCT Supervisor, } \\
\text { BWHC }\end{array}$ & FIC Initial/date \\
\hline $\begin{array}{l}\text { Verify Pin Tube } \\
\text { \& new overpack } \\
\text { identilications }\end{array}$ & $\begin{array}{l}\text { Proper } \\
\text { identification }\end{array}$ & N/A & 3M-TWP-98-017 & $\begin{array}{l}\text { HCT Supervisor, } \\
\text { BWHC }\end{array}$ & $\begin{array}{l}\text { HCT Initial/date } \\
\text { QC Initial/date }\end{array}$ \\
\hline $\begin{array}{l}\text { Place Pin Tube } \\
\text { in Overpack }\end{array}$ & $\begin{array}{l}\text { Ready for } \\
\text { transport in } \\
\text { SlRF Cask }\end{array}$ & $\mathrm{N} / \mathrm{A}$ & 3M-TWP-98-017 & $\begin{array}{l}\text { HCT Supervisor, } \\
\text { BWHC }\end{array}$ & Work plan steps \\
\hline $\begin{array}{l}\text { Transfer the Pin } \\
\text { Tube Overpack } \\
\text { from A-Cell to } \\
\text { SERF Cask }\end{array}$ & $\begin{array}{l}\text { Pin Tube } \\
\text { Overpack ready } \\
\text { for shipment }\end{array}$ & $\mathrm{N} / \mathrm{A}$ & $\begin{array}{l}\text { 3M-SOP-PTL- } \\
167,-118\end{array}$ & $\begin{array}{l}\text { HCT Supervisor, } \\
\text { BWHC }\end{array}$ & $\begin{array}{l}\text { Procedure steps, } \\
\mathrm{HCI} \text { signs } \\
\text { CFMI }\end{array}$ \\
\hline \multicolumn{6}{|c|}{327 A-Cell Pin Tube To SERF Cask (Shipment $C$ ) } \\
\hline $\begin{array}{l}\text { Verify A-Cell } \\
\text { Pin Tube \& new } \\
\text { overpack } \\
\text { identifications }\end{array}$ & $\begin{array}{l}\text { Proper } \\
\text { identification }\end{array}$ & $N / A$ & 3M-TWP-98-017 & $\begin{array}{l}\text { HCT Supervisor, } \\
\text { BWHC }\end{array}$ & $\begin{array}{l}\mathrm{HCl} \text { Initial/date } \\
\mathrm{QC} \text { Initial/date }\end{array}$ \\
\hline $\begin{array}{l}\text { Place Pin Tubc } \\
\text { in Overpack }\end{array}$ & $\begin{array}{l}\text { Ready for } \\
\text { transport in } \\
\text { SERF Cask }\end{array}$ & N/A & 3M-TWP-98-017 & $\begin{array}{l}\text { HCT Supervisor, } \\
\text { BWHC }\end{array}$ & Work plan steps \\
\hline $\begin{array}{l}\text { Transfer the Pin } \\
\text { Tube Overpack } \\
\text { from A-Cell to } \\
\text { SERF Cask }\end{array}$ & $\begin{array}{l}\text { Pin Tube } \\
\text { Overpack ready } \\
\text { for shipment }\end{array}$ & N/A & $\begin{array}{l}\text { 3M-SOP-PTI,- } \\
167,-118\end{array}$ & $\begin{array}{l}\text { HCT Supervisor, } \\
\text { BWHC }\end{array}$ & $\begin{array}{l}\text { Procedure steps, } \\
\text { HCT signs } \\
\text { CFMI } \\
\end{array}$ \\
\hline
\end{tabular}




\begin{tabular}{|c|c|c|c|c|c|}
\hline \multicolumn{6}{|c|}{ 327 SERF Cell Pin Tube To SERF Cask (Shipment C) } \\
\hline $\begin{array}{l}\text { Verity SERF } \\
\text { Cell Pin lube \& } \\
\text { ncu overpack } \\
\text { identilications }\end{array}$ & $\begin{array}{l}\text { Proper } \\
\text { identification }\end{array}$ & N/A & 3M-TWP-98-017 & $\begin{array}{l}\text { HCT Supervisor, } \\
\text { BWHC }\end{array}$ & $\begin{array}{l}\text { HCT Initial/date } \\
\text { QC Initial/date }\end{array}$ \\
\hline $\begin{array}{l}\text { Place Pin Tube } \\
\text { in Overpack }\end{array}$ & $\begin{array}{l}\text { Ready for } \\
\text { transport in } \\
\text { SERF Cask }\end{array}$ & N/A & 3M-TWP-98-017 & $\begin{array}{l}\text { HCT Supervisor, } \\
\text { BWHC }\end{array}$ & Work plan steps \\
\hline $\begin{array}{l}\text { Transfer the Pin } \\
\text { lube Overpack } \\
\text { from SERF Cell } \\
\text { to SERF Cask }\end{array}$ & $\begin{array}{l}\text { Pin Tube } \\
\text { Overpack ready } \\
\text { for shipment }\end{array}$ & N/A & $\begin{array}{l}\text { 3M-SOP-PTL- } \\
167,-118\end{array}$ & $\begin{array}{l}\text { HCT Supervisor, } \\
\text { BWHC }\end{array}$ & $\begin{array}{l}\text { Procedure steps, } \\
\text { HCT signs } \\
\text { CFMI }\end{array}$ \\
\hline \multicolumn{6}{|c|}{ Ship Pin Tubes From 327 To 324 D-Cell } \\
\hline $\begin{array}{l}\text { Ship from } 327 \text { to } \\
324\end{array}$ & $\begin{array}{l}\text { Transfer } \\
\text { complete } \\
\end{array}$ & $\mathrm{N} / \mathrm{A}$ & $\begin{array}{l}\text { 3M-SOP-PTL- } \\
167\end{array}$ & $\begin{array}{l}\text { HCT Supervisor, } \\
\text { BWHC }\end{array}$ & $\begin{array}{l}\text { MBA Custodian } \\
\& \text { QC signature }\end{array}$ \\
\hline $\begin{array}{l}324 \text { lacility } \\
\text { receive SERF } \\
\text { Cask fron } 327\end{array}$ & Ready to unload & $\mathrm{N} / \mathrm{A}$ & 3I-SOP-SMF-27 & $\begin{array}{l}\text { HCT Supervisor, } \\
\text { BWHC }\end{array}$ & $\begin{array}{l}\text { QC verify } \\
\text { shipping data } \\
\text { and CFMI }\end{array}$ \\
\hline $\begin{array}{l}\text { Unload SERI } \\
\text { Cask in REC } \\
\text { Airlock }\end{array}$ & $\begin{array}{l}\text { Ready for } \\
\text { loading into } \\
\text { cradle }\end{array}$ & N/A & $\begin{array}{l}\text { 3I-TWP-98-020, } \\
\text { 3I-SOP-SMF-27 }\end{array}$ & $\begin{array}{l}\text { HCT Supervisor, } \\
\text { BWHC }\end{array}$ & $\begin{array}{l}\text { MBA Custodian } \\
\text { Initial/date, } \\
\text { HCT signs } \\
\text { CFMI }\end{array}$ \\
\hline $\begin{array}{l}\text { Verify Pin Tube } \\
\text { Overpacks }\end{array}$ & $\begin{array}{l}\text { Proper } \\
\text { identification }\end{array}$ & N/A & 31-TWP-98-020 & $\begin{array}{l}\text { HCT Supervisor, } \\
\text { BWHC }\end{array}$ & $\begin{array}{l}\text { HCT Initial/date } \\
\text { QC Initial/date }\end{array}$ \\
\hline $\begin{array}{l}\text { Load Pin Tube } \\
\text { Overpacks into } \\
\text { lifting cradle }\end{array}$ & $\begin{array}{l}\text { Ready for } \\
\text { transfer to D- } \\
\text { Cell }\end{array}$ & N/A & 3I-TWP-98-020 & $\begin{array}{l}\text { HCT Supervisor, } \\
\text { BWHC }\end{array}$ & HCT Initial/date \\
\hline $\begin{array}{l}\text { Verity D-cell } \\
\text { storage location } \\
\text { \& lranster } \\
\text { matcrial }\end{array}$ & $\begin{array}{l}\text { Shipment } \mathrm{A} \text { to } \\
30 \text { gallon drum, } \\
\text { Shipments B\&C } \\
\text { to designated } \\
\text { D-Cell location }\end{array}$ & N/A & $\begin{array}{l}\text { 3I-TWP-98-020, } \\
\text { 3I-SOP-REC-G- } \\
\text { 2] }\end{array}$ & $\begin{array}{l}\text { HCT Supervisor, } \\
\text { BWHC }\end{array}$ & $\begin{array}{l}\text { MBA Custodian } \\
\text { initial/date, } \\
\text { HCT signs } \\
\text { CFM] } \\
\end{array}$ \\
\hline $\begin{array}{l}\text { I pdate and } \\
\text { verify criticality } \\
\text { postings }\end{array}$ & $\begin{array}{l}\text { Accurate } \\
\text { information }\end{array}$ & N/A & 3[-TWP-98-020 & $\begin{array}{l}\text { HCT Supervisor, } \\
\text { BWHC }\end{array}$ & $\begin{array}{l}\text { HCT Initial/date, } \\
\text { MBA Custodian } \\
\text { Litialdate }\end{array}$ \\
\hline
\end{tabular}




\section{DISTRIBUTION SHEET}

\begin{tabular}{|c|c|c|c|c|c|}
\hline \multirow{2}{*}{$\begin{array}{l}\text { To } \\
\text { Distribution }\end{array}$} & \multirow{2}{*}{\multicolumn{3}{|c|}{$\begin{array}{l}\text { From } \\
327 \text { Projects }\end{array}$}} & \multicolumn{2}{|l|}{ Page 1 of 1} \\
\hline & & & & \multicolumn{2}{|c|}{ Date $10-27-98$} \\
\hline \multirow{2}{*}{\multicolumn{4}{|c|}{$\begin{array}{l}\text { Project Title/Work Order } \\
327 \text { To } 324 \text { Pin Tube Shipment Quality Management Plan }\end{array}$}} & \multicolumn{2}{|c|}{ EDT No. 616491} \\
\hline & & & & \multicolumn{2}{|l|}{ ECN No. N/A } \\
\hline Name & MSIN & $\begin{array}{l}\text { Text } \\
\text { With All } \\
\text { Attach. }\end{array}$ & Text Only & $\begin{array}{l}\text { Attach./ } \\
\text { Appendix } \\
\text { Only }\end{array}$ & $\begin{array}{l}\text { EDT/ECN } \\
\text { Only }\end{array}$ \\
\hline
\end{tabular}

RP Bodeau

EJ Bitten

TL Erickson

JE Ham

KA Hedquist

DO Jenkins

SH Norton

DH Sandoz

RW Stevens

DS Takasumi

Central Files

D0E/RL Readina Room

\begin{tabular}{ll} 
LI -03 & $X$ \\
L1-02 & $X$ \\
L1-03 & $X$ \\
L1-08 & $X$ \\
L1-03 & $X$ \\
L1-01 & $X$ \\
L1-02 & $X$ \\
L1-06 & $X$ \\
L1-03 & $X$ \\
L1-03 & $X$ \\
B1-07 & $X$ \\
H2-53 & $X$ \\
\hline
\end{tabular}

